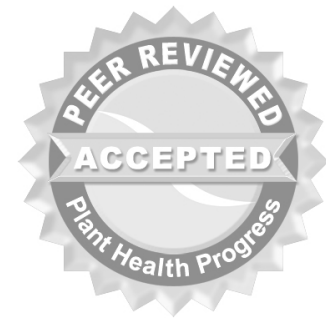

(c) 2008 Plant Management Network.

Accepted for publication 14 J uly 2008. Published 19 September 2008.

\title{
Postharvest Fruit Rots in Apples Caused by Botrytis cinerea, Phacidiopycnis washingtonensis, and Sphaeropsis py riputrescens
}

\author{
C. L. Xiao and Y. K. Kim, Tree Fruit Research and Extension Center, \\ Department of Plant Pathology, Washington State University, 1100 \\ North Western Avenue, Wenatchee, WA 98801
}

Corresponding author: C. L. Xiao.clxiao@wsu.edu

Xiao, C. L., and Kim, Y. K. 2008. Postharvest fruit rots in apples caused by Botrytis cinerea, Phacidiopycnis washingtonensis, and Sphaeropsis pyriputrescens. Online. Plant Health Progress doi: 10.1094/PHP-2008-0919-01-DG.

\section{I ntroduction}

After harvest, apple fruit may be stored in cold storage for several months or up to 12 months in controlled-atmosphere (low $\mathrm{O}_{2}$ and high $\mathrm{CO}_{2}$ ) storage prior to packing. Packed fruit may also be held in cold storage for an extended period of time before shipping to the market, depending on the market needs. Postharvest fruit rot diseases can be a limiting factor for storage of apples. Gray mold caused by Botrytis cinerea is a common postharvest disease of apples worldwide (12). Sphaeropsis rot caused by the fungus Sphaeropsis pyriputrescens and speck rot caused by the fungus Phacidiopycnis washingtonensis are two recently reported postharvest fruit rot diseases of apples in the United States $(6,16,17,18)$. Symptoms of these three diseases can appear on infected fruit as early as within three months in cold storage after harvest, but most decays that originate from infections of the stem and calyx of fruit develop after an extended period of time in storage. In a recent survey of postharvest diseases of apples conducted on cultivars Red Delicious, Fuji, and Golden Delicious in Washington State, gray mold and Sphaeropsis rot account for $28 \%$ and $17 \%$ of the total decay, respectively (7). Speck rot occurred sporadically in Washington State during the survey from 2003 to 2005 (6). However, both Sphaeropsis rot and speck rot can cause significant economic losses of apple fruit during storage. For example, instances with up to $24 \%$ losses resulting from Sphaeropsis rot or speck rot have been observed on Red Delicious apples during storage (Figs. 1 and 2).

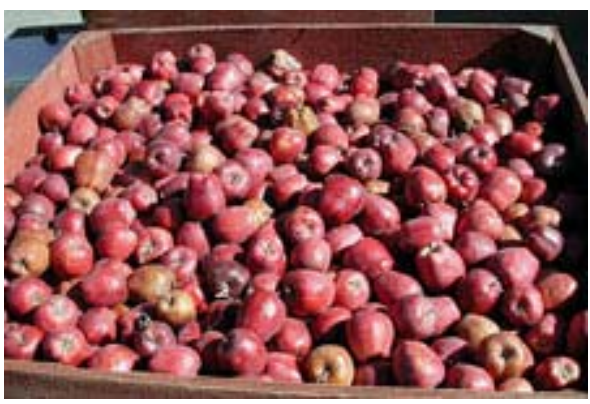

Fig. 1. In this grower lot of Red Delicious from a commercial fruit packinghouse, approximately $24 \%$ of the apple fruit were decayed by Sphaeropsis pyriputrescens.

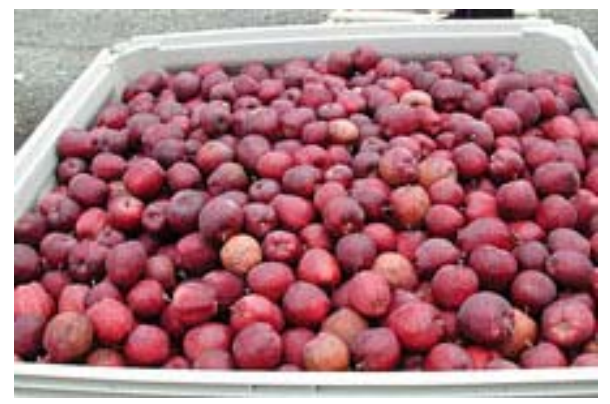

Fig. 2. In this grower lot of Red Delicious from a commercial fruit packinghouse, approximately $24 \%$ of the apple fruit were decayed by Phacidiopycnis washingtonensis. 
Identifying target pathogens is the first necessary step for development and implementation of relevant measures for disease control. However, the symptoms of these two new diseases are similar to those of gray mold, and the two newly recognized diseases are often misdiagnosed as gray mold. The objective of this article is to provide a practical guide to diagnosis of these three diseases in apples.

Hosts: Apple (Malus × domestica Borkh.).

Diseases: Gray mold; speck rot; and Sphaeropsis rot.

Pathogens: Botrytis cinerea Pers., teleomorph Botryotinia fuckeliana (de Bary) Whetzel (3); Phacidiopycnis washingtonensis Xiao \&J.D. Rogers (18); and Sphaeropsis pyriputrescens Xiao \&J .D. Rogers (16).

\section{Taxonomy}

Botrytis cinerea is the anamorph of the discomycete Botryotinia fuckeliana (de Bary) Whetzel (3), which is placed in Sclerotiniaceae of Helotiales (9). Most strains are heterothallic (1). The teleomorph state is seldom found in nature (10), but apothecia can be readily obtained in the laboratory (4).

Phacidiopycnis washingtonensis was described in 2005 by Xiao et al. (18). It is a pycnidial anamorphic fungus. Currently, there are five species in the genus Phacidiopycnis (18). The form-genus Phacidiopycnis was established by Potebnia (11) on P. malorum, now P. piri (2). All Phacidiopycnis species have teleomorphs referable to Potebniamyces Smerlis (2), which is placed in Rhytismataceae of Rhytismatales (9). The teleomorph state of P. washingtonensis was not found based on a small-scale orchard survey in 2003 (18).

Sphaeropsis pyriputrescens was described in 2004 by Xiao and Rogers (16). It is a pycnidial anamorphic fungus. The teleomorph of the fungus has not been reported.

\section{Symptoms and Signs}

Gray mold. Gray mold originates primarily from infection of wounds such as cracks at the stem-bowl area of Gala apple fruit (Fig. 3) and punctures and bruises that are created at harvest and during postharvest handling. Stem-end gray mold (Figs. 4 and 5) and calyx-end gray mold (Figs. 6 and 7) can also occur on apples. Calyx-end gray mold likely originates from infections of floral parts of fruit, but this type of gray mold symptom is not commonly seen on apples grown in a semi-arid climate such as that of eastern Washington State. Gray mold can also originate from fruit-to-fruit spread of the disease in field bins during storage (Fig. 8). Depending on apple cultivar and storage conditions, the decayed area appears light brown to dark brown (Figs. 3 to 9). When the entire fruit is decayed, it may appear like a baked apple. The decayed area is spongy and diseased tissue is not readily separable from the healthy tissue, but after an extended storage, advanced decayed fruit may become soft. Under high relative humidity, grayish spore masses and/ or fluffy white to gray mycelium may appear on the decayed area (Figs. 5, 7, and 9). Except that a mild cider-like odor may be associated with gray mold decayed apple fruit, generally there is no detectable distinct odor from gray mold decayed fruit. 


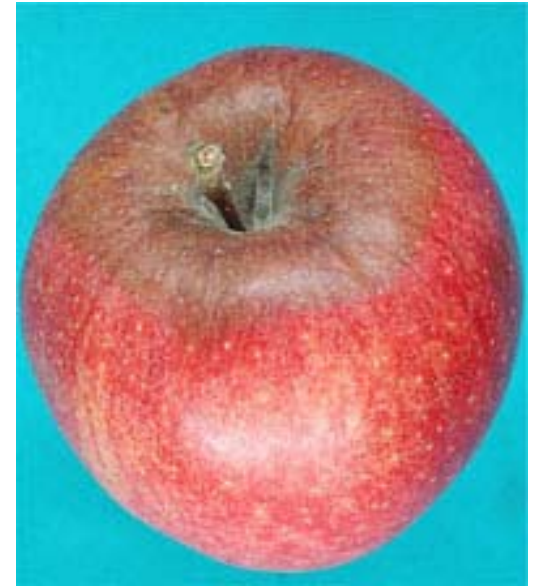

Fig. 3. Gray mold originating from infection of stem bowl crack of a Gala apple.

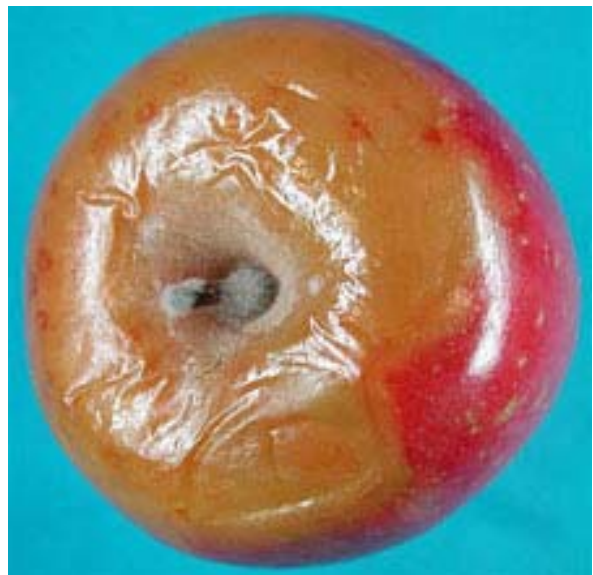

Fig. 5. Stem-end gray mold on a Fuji fruit showing gray spore masses on the stem.

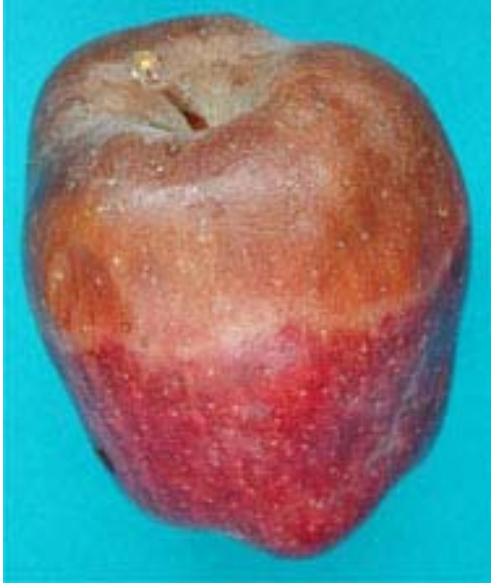

Fig. 4. Gray mold originating from infection of the stem of a Red Delicious apple fruit, resulting in stem-end rot.

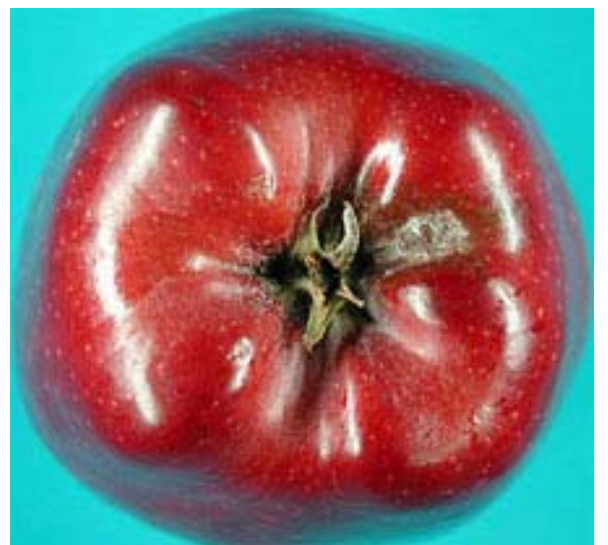

Fig. 6. Early stage of calyx-end gray mold on a Red delicious apple fruit.

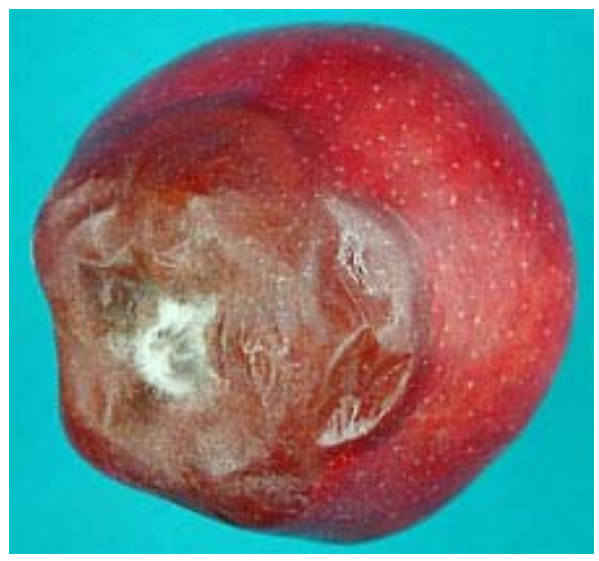

Fig. 7. Calyx-end gray mold on a Red Delicious fruit showing white to gray mycelium in the calyx area. 


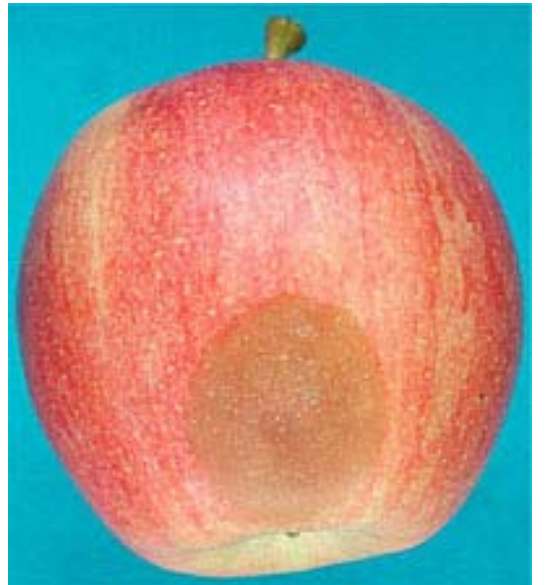

Fig. 8. Early stage of gray mold resulting from fruit-to-fruit spread of gray mold.

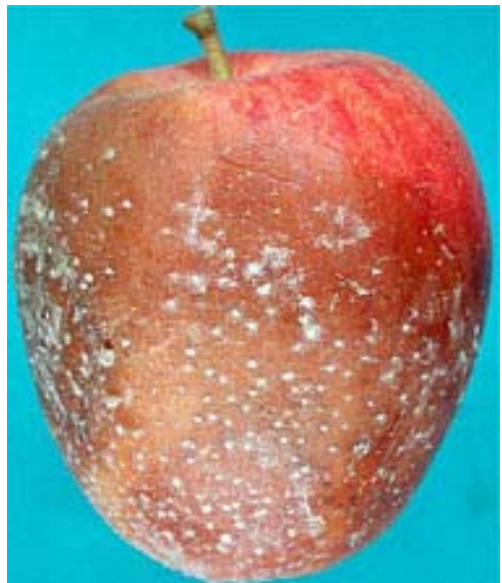

Fig. 9. Advanced stage of gray mold showing white to gray mycelium and gray spore masses of Botrytis cinerea on gray mold decayed fruit under high relative humidity.

Speck rot. Stem-end rot (Figs. 10 and 11) and calyx-end rot (Figs. 12 to 15) are the two major types of symptoms of speck rot on apple fruit. The fungus colonizes the stem and calyx of the fruit and symptoms advance along the vascular tissues in the fruit (Figs. 16 and 17). The decayed tissue is spongy to firm and not readily separable from the healthy tissue. The color of the decayed area varies from light brown to dark brown or occasionally black (Figs. 10 to 15 and 18 to 20). On red apple cultivars particularly, brown to black specks with a white to light tan center around lenticels often appear on speck rot decayed fruit as the disease advances (Figs. 10 to 13). As a result, the peel color of entire decayed fruit may appear light brown, brown, or pink (discolored from red) with specks covering a part or the entire fruit (Figs. 18 to 20). These brown to black specks are a typical characteristic of speck rot. The peel color on aged decayed areas may turn black and the fungus may form pycnidia on the surface of the decayed area, starting from the infection sites, after the fruit have been stored for an extended period of time (Fig. 15). Speck rot decayed fruit produce a mild distinct odor in advanced stages.

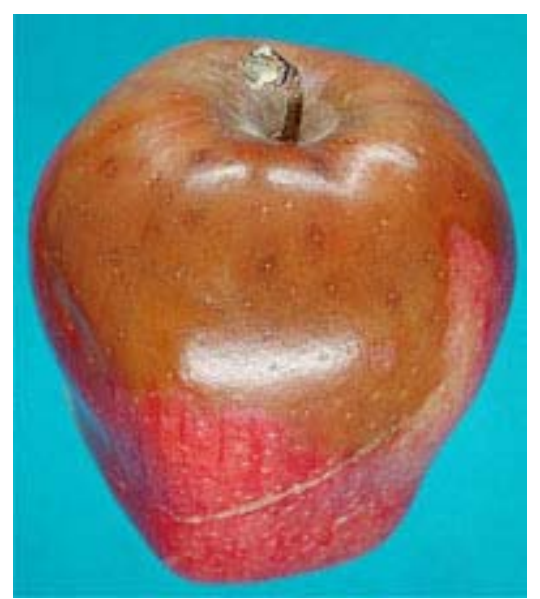

Fig. 10. Speck rot originating from infection of the stem of Red delicious fruit, resulting in stem-end rot.

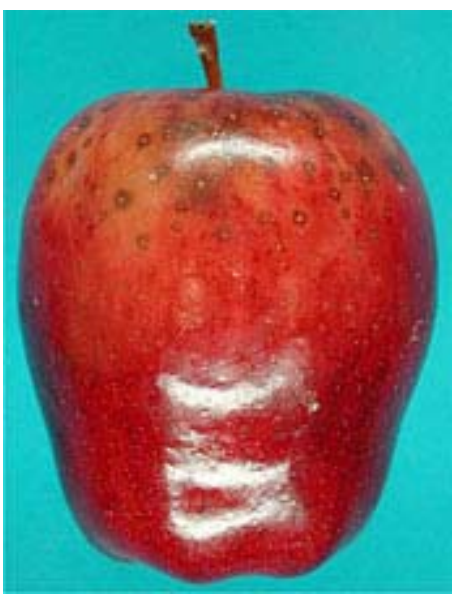

Fig. 11. Stem-end speck rot showing brown to black specks around the lenticels of the surface of decayed area. 


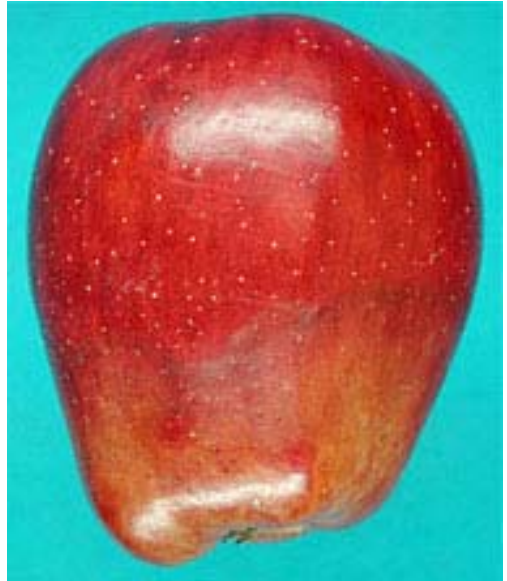

Fig. 12. Speck rot originating from infection of the calyx tissue of apple fruit, resulting in calyx-end rot.

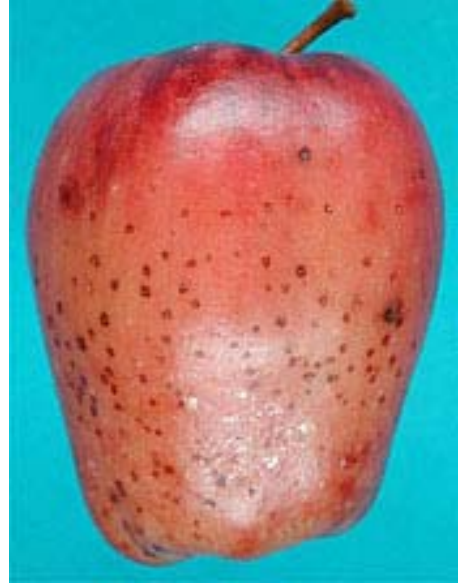

Fig. 13. Calyx-end speck rot showing brown to black specks around the lenticels as the disease advacnes.

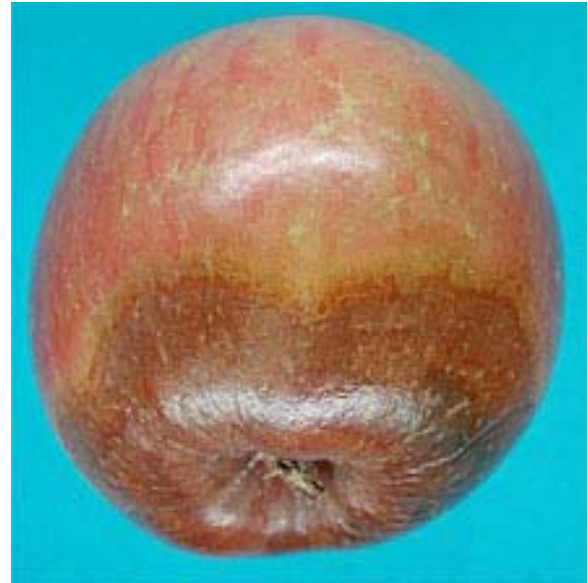

Fig. 14. Calyx-end speck rot on a Fuji apple showing aging area starting to turn darker brown.

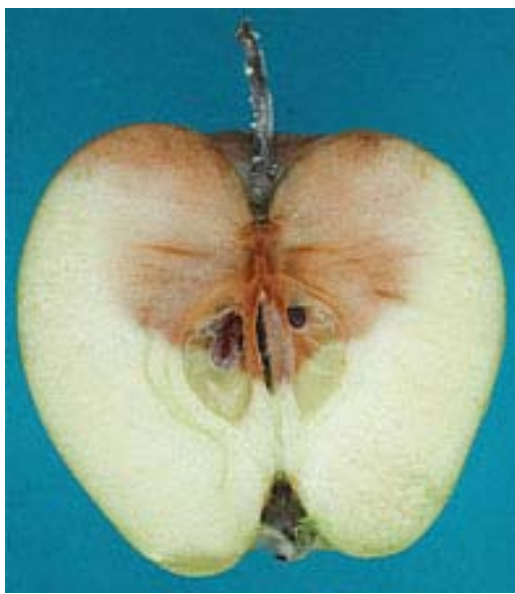

Fig. 16. Internal symptoms of stemend speck rot showing the disease advancing along the vascular tissue in the fruit.

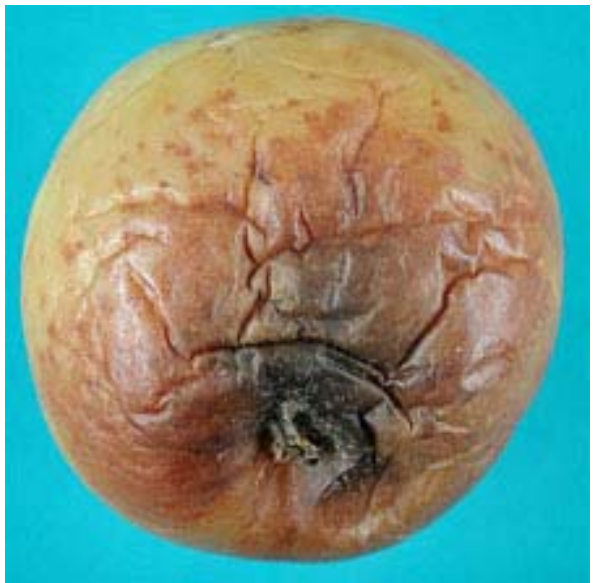

Fig. 15. Advanced stage of speck rot on a Fuji apple. The aged decayed area turns dark brown to black after an extended storage period. Pycnidia of Phacidiopycnis washingtonensis may form on the decayed area, starting from the infection site.

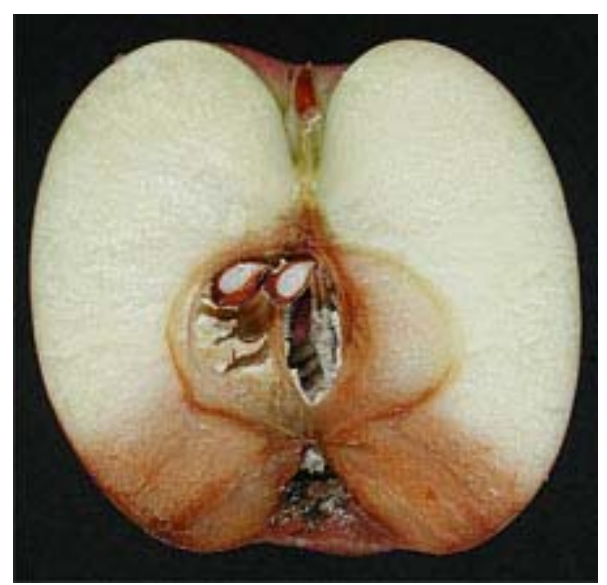

Fig. 17. Internal symptoms of calyx-end speck rot showing the disease advancing along the vascular tissue in the fruit. 


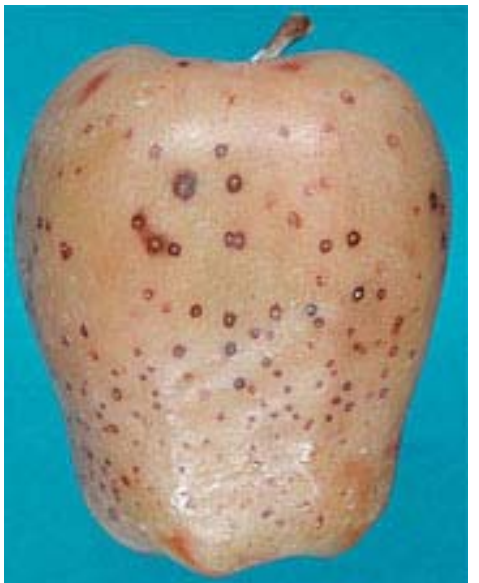

Fig. 18. Advanced stage of speck rot on a Red delicious fruit showing discolored fruit with brown to black specks around the lenticels.

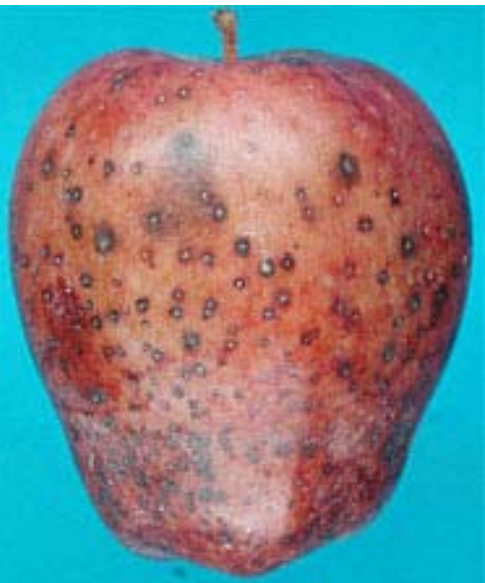

Fig. 19. Advanced stage of speck rot on a Red delicious fruit showing pink appearance with brown to black specks around the lenticels.

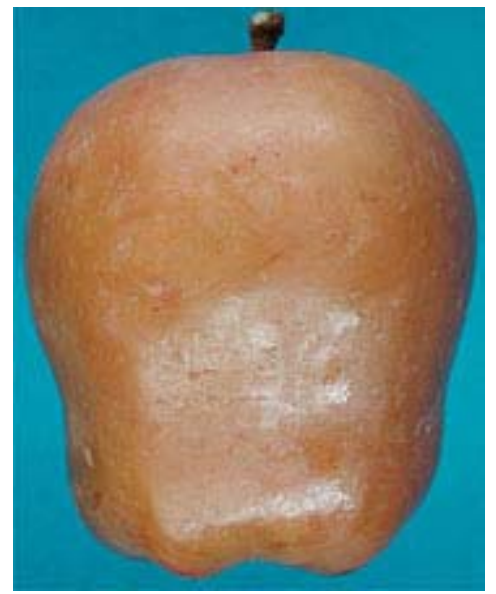

Fig. 20. Advanced stage of speck rot on a Red delicious fruit showing discolored fruit with very few specks around the lenticels.

The other type of symptom of speck rot on apple is core rot, which likely results from infections of floral parts of the fruit in the early fruit-growing season. This type of speck rot was not observed during the survey of apple postharvest diseases conducted during 2003 through 2005 (6), but an instance of considerable losses of Fuji apple fruit due to rots was reported to us in March 2008 by a fruit packinghouse. In this case, speck rot was responsible for the losses of apple fruit in storage and in addition to stem-end and calyx-end speck rot, a core rot caused by P. washingtonensis also was observed and confirmed by isolation of the causal agent (Xiao and Kim, personal observation). In the early stage of core rot caused by P. washingtonensis, external symptoms are not present. In the advanced stage, external symptoms of core rot may be first seen at the stem-bowl or calyx-end area, or as irregular decay lesions on the fruit surface. When the fruit is cut, it is evident that the disease advances starting at the core area from inside the fruit outwards (Fig. 21). 

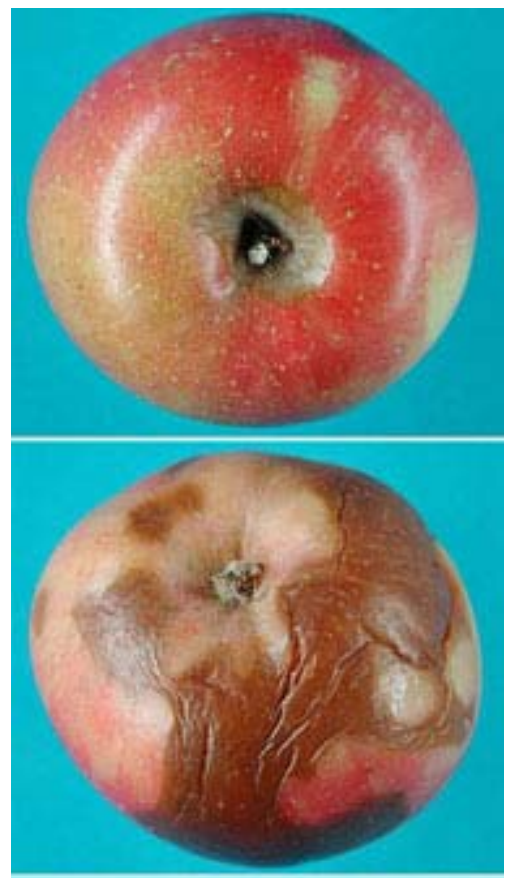

Fig. 21.

A core rot on Fuji apple caused by Phacidiopycnis washingtonensis. External symptoms of core rot may be seen at the stem-bowl area (top) and as irregular lesions on the fruit surface (center), but disease starts from the core area and advances from inside outwards (bottom).

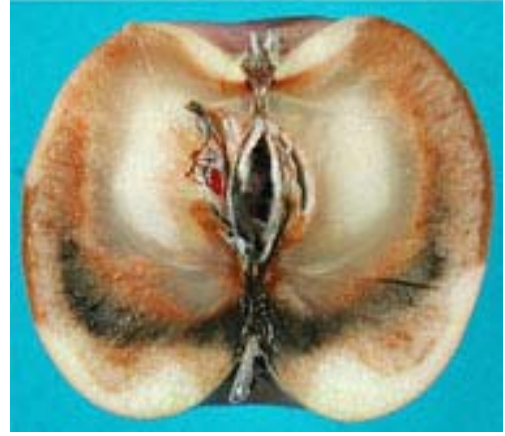

Sphaeropsis rot. Stem-end rot (Figs. 22 to 24) and calyx-end rot (Figs. 25 to 28) are the two major types of symptoms of Sphaeropsis rot on apples.

Sphaeropsis rot can also originate from infection of lenticels of fruit (Fig. 29). Infection of lenticels is more commonly found on Golden Delicious fruit than on Fuji and Red Delicious fruit, but this type of symptom generally is less common. The decayed tissues are firm and not separable from the healthy tissues. S. pyriputrescens infects the stem and calyx of the fruit and advances along the vascular tissues of the fruit (Fig. 30). The color of decayed areas is light brown to brown and may turn darker in the aged decayed area (Figs. 22 to 29). As the disease advances, the peel color of the entire decayed fruit may be similar or vary across the fruit surface (Figs. 31 and 32 ). In the advanced stage, pycnidia of the fungus may form on the surface of decayed fruit. The pycnidia are black, superficial or partially embedded in the decayed tissue (Fig. 33). Fruit decays caused by Sphaeropsis rot have a distinct "bandage-like" odor, particularly in the decayed flesh when the fruit is cut. 


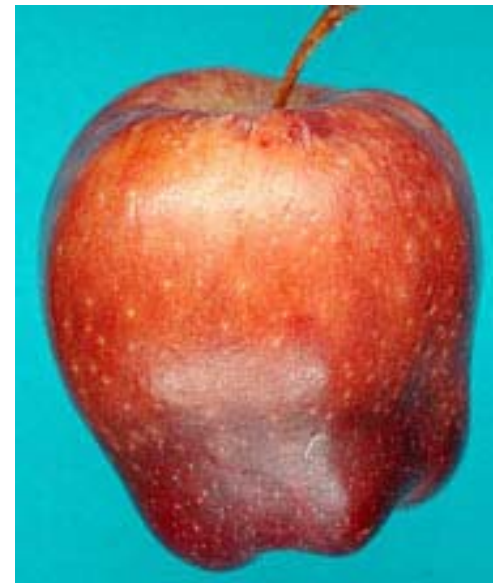

Fig. 22. Stem-end Sphaeropsis rot originating from infection of the stem of a Red Delicious fruit.

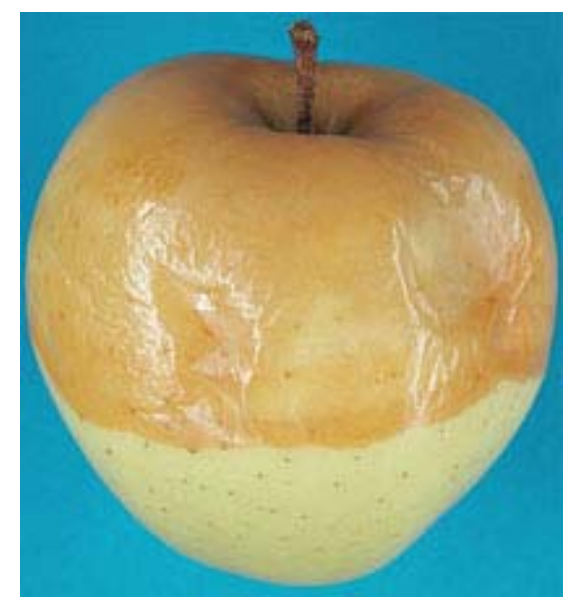

Fig. 24. Stem-end Sphaeropsis rot on a Golden Delicious fruit.

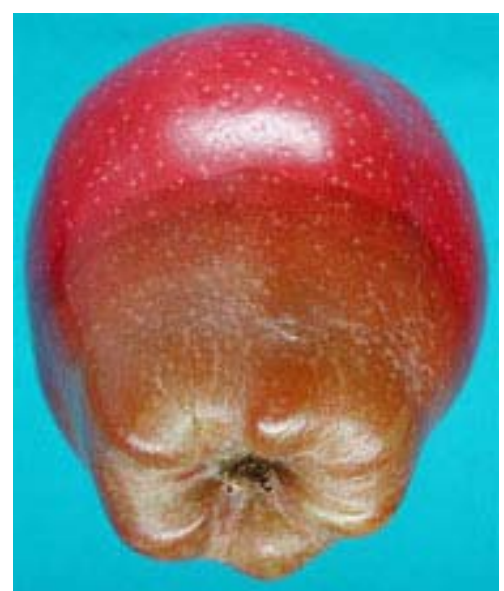

Fig. 26. Calyx-end Sphaeropsis rot on a Red Delicious apple fruit.

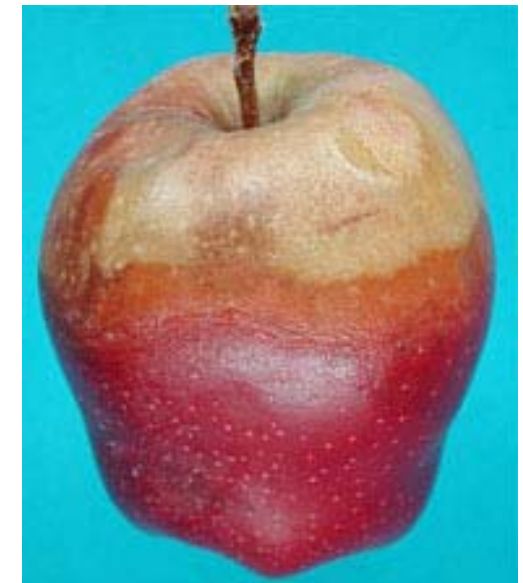

Fig. 23. Stem-end Sphaeropsis rot showing that varying color may appear across the decayed area.

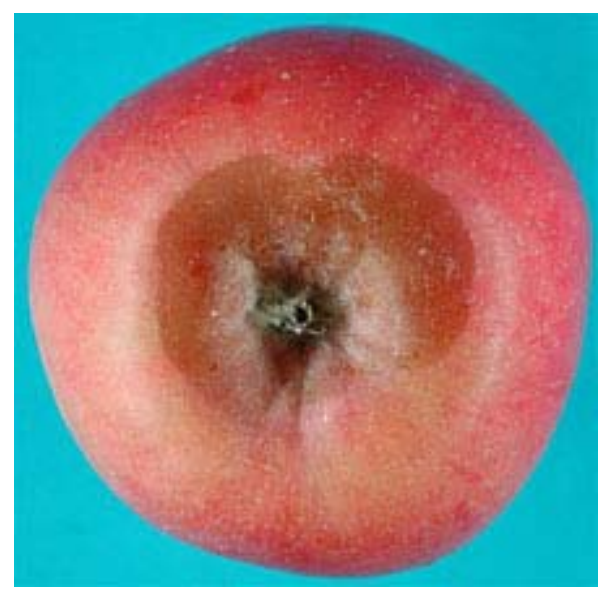

Fig. 25. Early stage of calyx-end

Sphaeropsis rot originating from infection of the calyx tissue of a Fuji apple fruit.

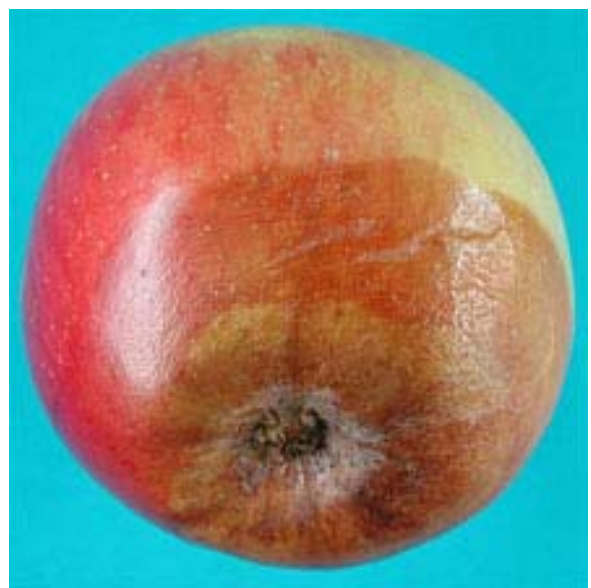

Fig. 27. Calyx-end Sphaeropsis rot showing that aging decayed area turns dark brown and pycnidia of the fungus forms on the decayed area, starting from the infection site. 


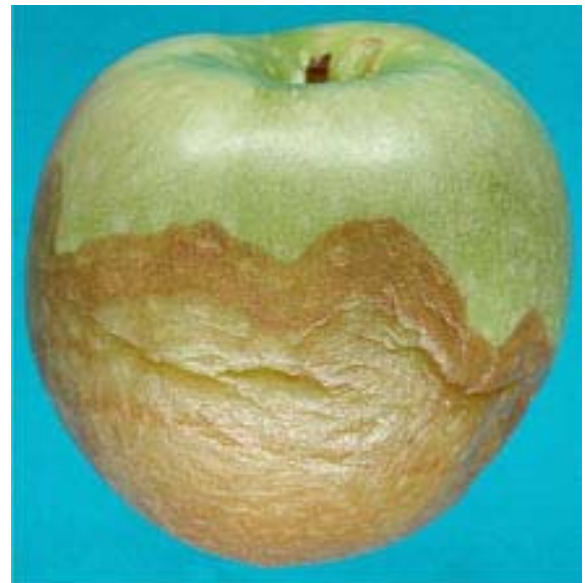

Fig. 28. Calyx-end Sphaeropsis rot on a Granny Smith apple fruit showing lesion with an irregular margin.

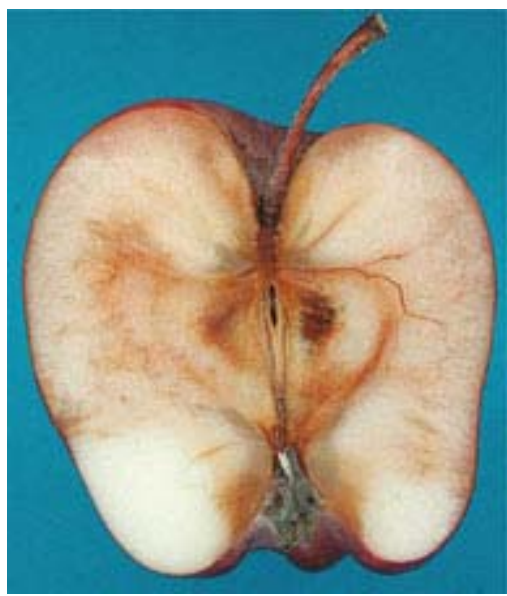

Fig. 30. Internal symptoms of stemend Sphaeropsis rot on a Red

Delicious apple fruit showing that the disease advances along the vascular tissue in the fruit.

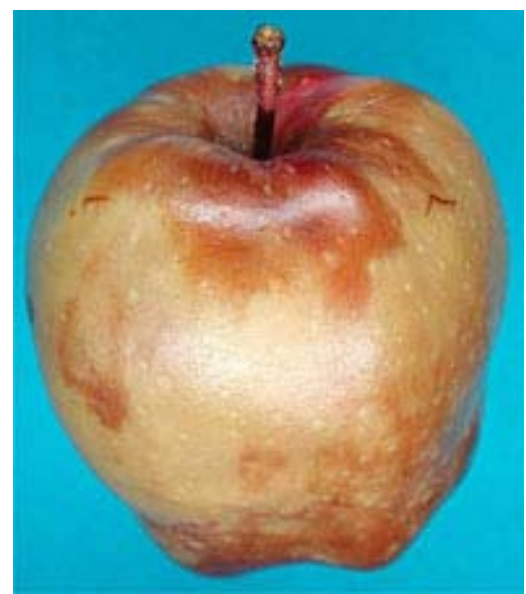

Fig. 32. An entire Red Delicious apple fruit decayed by Sphaeropsis rot showing that the color of decayed area may vary across the fruit surface.

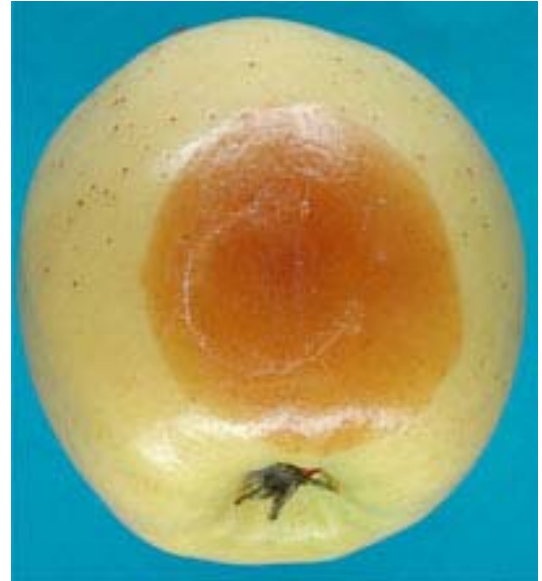

Fig. 29. Sphaeropsis rot originating from infection of lenticels on a Golden Delicious apple fruit.

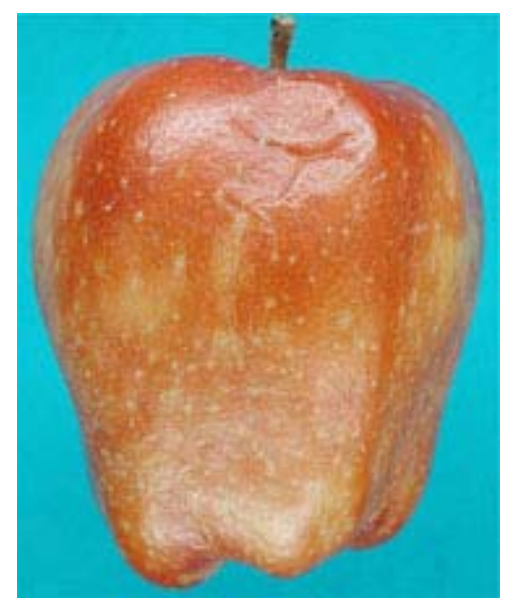

Fig. 31. An entire Red Delicious apple fruit decayed by Sphaeropsis rot turns brown.

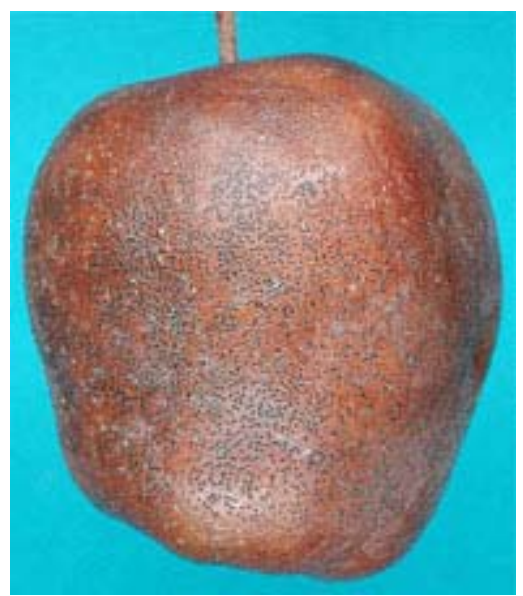

Fig. 33. Pycnidia of Sphaeropsis pyriputrescens form on a decayed Red Delicious apple fruit after an extended period of time in storage. 


\section{Host Range}

B. cinerea causes diseases on many plant species, including Malus $\times$ domestica $(3,5)$. P. washingtonensis has been reported only on Malus $\times$ domestica Borkh., Malus spp. (crabapple), and Pyrus communis $(6,18)$. S. pyriputrescens has been reported on Malus $\times$ domestica, Malus spp. (crabapple), and Pyrus communis $(15,16,17)$. It is not known whether S. pyriputrescens also infects Asian pear species.

\section{Geographical Distribution}

B. cinerea is widespread in the world (3). P. washingtonensis has been reported in Washington State of the United States $(6,18)$. S. pyriputrescens has been reported on apple and pear in Washington State $(15,16,17)$ and on pear in British Columbia, Canada (13).

\section{Pathogen I solation}

The three fungi can be readily isolated from diseased fruit. To isolate these fungi, decayed fruit are sprayed with $70 \%$ ethanol and air-dried. The fruit peel at the margin of decayed and healthy tissues is removed with a sterile scalpel, and small pieces of the decayed fruit flesh are excised and plated on acidified potato dextrose agar (APDA; Difco Laboratories, Detroit, MI) $(4.0 \mathrm{ml}$ of a $25 \%$ solution of lactic acid per liter of medium). Plates are incubated at room temperature (20 to $22^{\circ} \mathrm{C}$ ) for 3 to 14 days and examined for culture development.

\section{Pathogen I dentification}

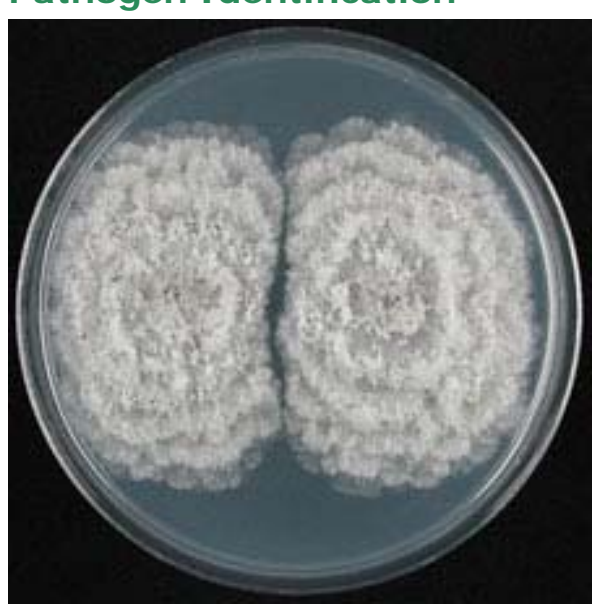

Fig. 34. A 7-day-old culture of Phacidiopycnis washingtonensis on an isolation plate incubated at $20^{\circ} \mathrm{C}$ in the dark.

pycnidia of the fungus (8).

Botry tis cinerea. Isolates of B. cinerea from decayed apple fruit or decayed pear fruit vary in colony morphology, sporulation, and formation of sclerotia on PDA. Descriptions and illustrations of morphology of B. cinerea isolates from decayed pear fruit have been published and can also be used for aid in identification of the fungus from apple (14).

Phacidiopy cnis washingtonensis. On isolation plates incubated at $20^{\circ} \mathrm{C}$ in the dark, colonies are light gray to gray with fluffy aerial mycelium. Alternating rings of gray and dark gray become evident, particularly on the reverse of the plate, after the cultures have been incubated for 7 days (Fig. 34, above, and Figs. 35 to 37). Formation of pycnidia often can be seen on 7-day-old PDA cultures (Figs. 34 and 36). Abundant pycnidia and sporulation of pycnidia (creamcolored oozing of conidia from pycnidia) are often present on 14-day-old PDA cultures (Fig. 38). P. washingtonensis can also readily grow on other agar media. Growth characteristics of P. washingtonensis on various agar media 
were described by Xiao et al. (18). Conidia of P. washingtonensis are hyaline, lacriform with the dehiscence end flattened or ovoid to ellipsoid without the cicatrice, smooth, (4.4-) 5.9-7.3 (-8.8) ×2.2-3.7 (-5) pm (Fig. 39).

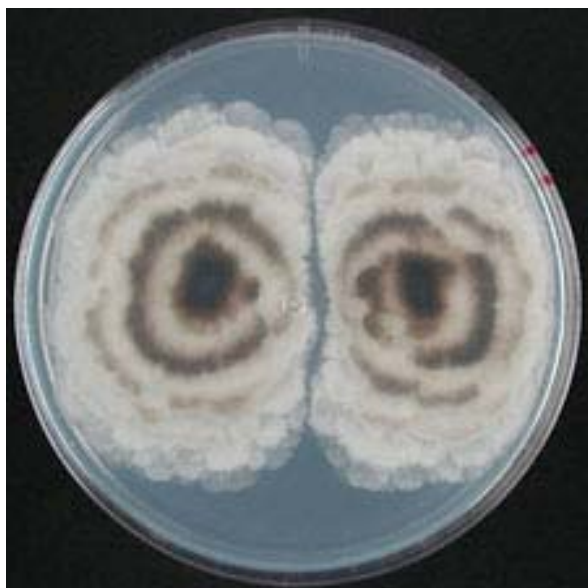

Fig. 35. The reverse of a 7-day-old culture of Phacidiopycnis washingtonensis on an isolation plate incubated at $20^{\circ} \mathrm{C}$ in the dark showing gray and dark gray alternating rings.

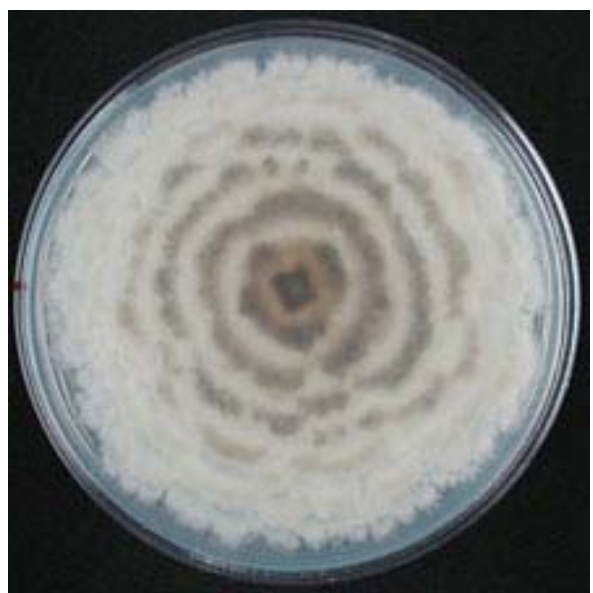

Fig. 37. The reverse of a 10-day-old culture of Phacidiopycnis washingtonensis on potato dextrose agar incubated at $20^{\circ} \mathrm{C}$ in the dark.

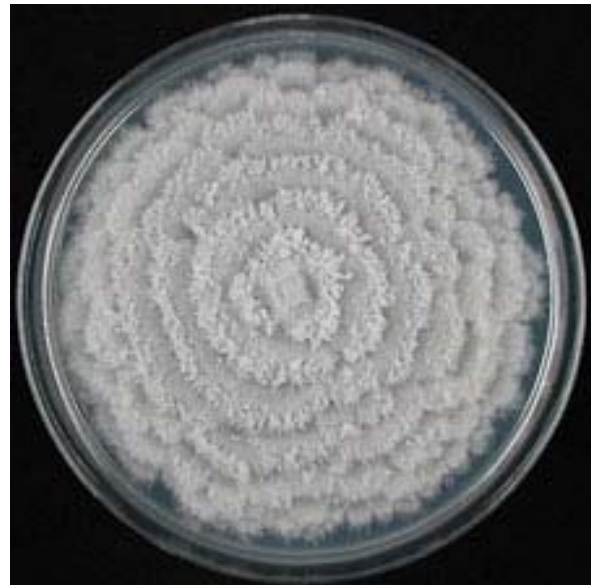

Fig. 36. A 10-day-old culture of Phacidiopycnis washingtonensis on potato dextrose agar incubated at $20^{\circ} \mathrm{C}$ in the dark.

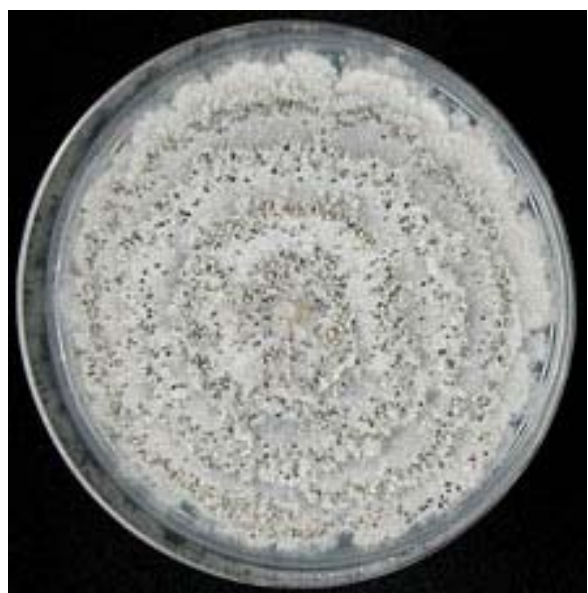

Fig. 38. A 14-day-old potato dextrose agar culture of Phacidiopycnis washingtonensis incubated at $20^{\circ} \mathrm{C}$ in the dark showing pycnidia and sporulation of pycnidia (cream-colored oozing of conidia from pycnidia).

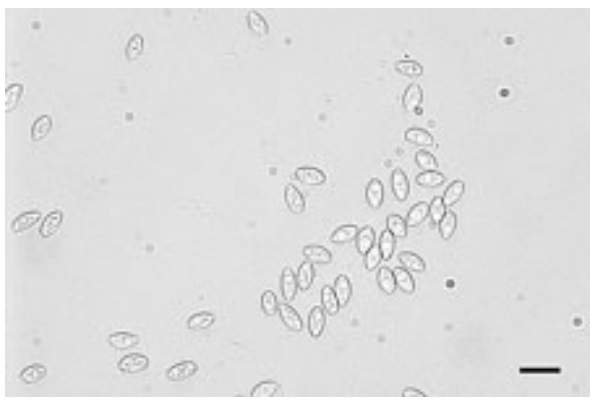

Fig. 39. Conidia of Phacidiopycnis washingtonensis. Scale bar $=10 \mu \mathrm{m}$.

Sphaeropsis pyriputrescens. Descriptions and illustrations of morphology of S. pyriputrescens isolates from decayed pear fruit have been published (14). 
Growth characteristics of S. pyriputrescens on various agar media were described by Kim et al. (8). Unlike B. cinerea and P. washingtonensis, one distinct characteristic of S. pyriputrescens is the presence of yellow pigmentation of colonies on PDA. This yellow pigmentation of colonies on PDA is a very useful characteristic in the identification of this fungus.

\section{Pathogen Storage}

Mycelium plugs of PDA cultures of B. cinerea, P. washingtonensis, and S. pyriputrescens can be stored either in sterile water at $4^{\circ} \mathrm{C}$ for short-term storage (within 6 months) or in $15 \%$ glycerol at $-80^{\circ} \mathrm{C}$ for long-term storage.

\section{Pathogenicity Tests}

The following methods have been proven successful in testing pathogenicity of these fungi on apple fruit.

The conidial states of the three fungi are the main type of inoculum responsible for fruit infection. In pathogenicity tests, conidia of B. cinerea produced on 10- to 14-day-old PDA cultures, pycnidia of P. wahingtonensis produced on 2-week-old PDA cultures, and pycnidia of S. pyriputrescens produced on 3-week-old OMA cultures at $20^{\circ} \mathrm{C}$ under 12 -h alternating cycles of dark and fluorescent light are used to make conidial suspensions for fruit inoculation. Concentrations of conidial suspensions are adjusted to $1 \times 10^{4}$, $1 \times 10^{5}$ and $1 \times 10^{4}$ conidia/ $\mathrm{ml}$ for B. cinerea, P. washingtonensis, and S. pyriputrescens, respectively.

To avoid potential interference of fungicide residues on pathogenicity tests, fruit that are harvested from orchards neither sprayed with fungicides within 2 to 4 weeks before harvest nor treated with postharvest fungicides are used for pathogenicity tests in the laboratory. Prior to inoculation, fruit are surfacedisinfested for $5 \mathrm{~min}$ in $0.5 \% \mathrm{NaOCl}$, rinsed three times with sterile water, and then air-dried.

To test pathogenicity of B. cinerea, fruit are wounded with a 4-mm-diameter finishing nail head to a depth of $4 \mathrm{~mm}$ and then inoculated by placing $20 \mu \mathrm{l}$ of conidial suspensions at each wound with a pipette. Inoculated fruit are placed on fiberboard apple trays wrapped in perforated polyethylene liners and stored in cardboard boxes in air at $0^{\circ} \mathrm{C}$. Decay development is evaluated 6 to 8 weeks after inoculation.

Pathogenicity tests for P. washingtonensis and S. pyriputrescens can be done either in the laboratory or in the orchard. To inoculate the stem and calyx of apple fruit in the laboratory, the end of the stem of each fruit is slightly cut horizontally with a sterile scalpel to make a wound. The end of the stem and calyx are inoculated by covering them with a piece of wet cheesecloth $(2 \times 2 \mathrm{~cm})$, which has been dipped into the conidial suspensions of either pathogen prior to inoculation. Fruit treated with cheesecloth moistened with sterile water are used as noninoculated controls. To facilitate infections at the stem and calyx, inoculated fruit are kept in aluminum muffin trays in plastic containers with a shallow depth of water on the bottom at room temperature $\left(20\right.$ to $\left.22^{\circ} \mathrm{C}\right)$ overnight. Cheesecloth is then removed and fruit are stored in air at $0^{\circ} \mathrm{C}$. Stemend rot and calyx-end rot are evaluated 2 to 3 months after inoculation.

To test pathogenicity of $\mathrm{P}$. washingtonensis and $\mathrm{S}$. pyriputrescens on apple fruit in the orchard, fruit on replicate trees are inoculated 1 to 2 weeks before harvest with conidial suspensions of either pathogen using a hand sprayer until runoff. Control fruit are sprayed with sterile water. The fruit are then covered with moistened plastic bags to maintain high humidity. Plastic bags are covered with white paper bags to prevent sunlight from generating damaging heat on fruit. Preferred timing for inoculations is near sunset. After approximately $15 \mathrm{~h}$ of incubation, the bags are removed. All fruit are harvested, transferred onto fiber foam apple-trays wrapped in perforated polyethylene bags, and stored in cardboard boxes in air at $0^{\circ} \mathrm{C}$. Development of stem-end rot and calyx-end rot on the fruit are monitored monthly for up to 7 months, starting 2 months after harvest. 


\section{Acknowledgments}

This manuscript is Plant Pathology New Series 0492, Department of Plant Pathology, College of Agricultural, Human, and Natural Resource Sciences Agricultural Research Center, Project No. WNP00367, Washington State University, Pullman, WA 99164-6430.

\section{Literature Cited}

1. Beever, R. E., and Weeds, P. L. 2004. Taxonomy and genetic variation of Botrytis and Botryotinia. Pages 29-52 in: Botrytis: Biology, Pathology and Control. Y. Elad, B. Williamson, P. Tudzynski, and N. Delen, eds. Kluwer Academic Publ., Dordrecht, The Netherlands.

2. DiCosmo, F., Nag Raj, T. R., and Kendrick, W. B. 1984. A revision of the Phacidiaceae and related anamorphs. Mycotaxon 21:1-234.

3. Coley-Smith, J. R., Verhoeff, K., and J arvis, W. R. 1980. The Biology of Botrytis. Academic Press, San Diego, CA.

4. Faretra, F., Antonacci, E., and Pollastro, S. 1988. Sexual behaviour and mating system of Botryotinia fuckeliana, teleomorph of Botrytis cinerea. J . Gen. Microbiol. 134:2543-2550.

5. Farr, D. F., Bills, G. F., Chamuris, G. P., and Rossman, A. Y. 1989. Fungi on Plants and Plant Products in the United States. American Phytopathological Society, St. Paul, MN.

6. Kim, Y. K., and Xiao, C. L. 2006. A postharvest fruit rot in apple caused by Phacidiopycnis washingtonensis. Plant Dis. 90:1376-1381.

7. Kim, Y. K., and Xiao, C. L. 2008. Distribution and incidence of Sphaeropsis rot in apple in Washington State. Plant Dis. 92:940-946.

8. Kim, Y. K., Xiao, C. L., and Rogers, J. D. 2005. Influence of culture media and environmental factors on mycelial growth and pycnidial production of Sphaeropsis pyriputrescens. Mycologia 97:25-32.

9. Kirk, P. M., Cannon, P. F., David, J . C., and Stalpers, J . A. 2001. Ainsworth and Bisby's Dictionary of the Fungi. 9th ed. CAB Intl., Wallingford, UK.

10. Lorbeer, J. W. 1980. Variation in Botrytis and Botryotinia. Pages 19-40 in: The Biology of Botrytis. J. R. Coley-Smith, K. Verhoeff, and W. R. J arvis, eds. Academic Press, San Diego, CA.

11. Potebnia, A. 1912. Ein neuer Krebserreger des Apfelbaumes Phacidiella discolor (Mout. Et Sacc.) A. Pot., seine Morphologie und Entwickelungsgeschichte. Z. Pflanz. 22:129-153.

12. Rosenberger, D. A. 1990. Gray mold. Pages 55-56 in: Compendium of Apple and Pear Diseases. A. L. J ones and H. S. Aldwinckle, eds. American Phytopathological Society, St. Paul, MN.

13. Stokes, S. C., Sholberg, P. L., O'Gorman, D. T. 2007. Two new pathogens of pear, Phacidiopycnis pyri and Sphaeropsis pyriputrescens, identified for the first time in British Columbia. (Abstr.) Can. J. Plant Pathol. 29:86.

14. Xiao, C. L. 2006. Postharvest fruit rots in d'Anjou pears caused by Botrytis cinerea, Potebniamyces pyri, and Sphaeropsis pyriputrescens. Online. Plant Health Progress doi:10.1094/ PHP-2006-0905-01-DG.

15. Xiao, C. L., and Boal, R. J . 2005. A new canker and twig dieback disease of apple and crabapple trees caused by Sphaeropsis pyriputrescens in Washington State. Plant Dis. 89:1130.

16. Xiao, C. L., and Rogers, J. D. 2004. A postharvest fruit rot in d'Anjou pears caused by Sphaeropsis pyriputrescens sp. nov. Plant Dis. 88:114-118.

17. Xiao, C. L., Rogers, J. D., and Boal, R. J. 2004. First report of a new postharvest fruit rot on apple caused by Sphaeropsis pyriputrescens. Plant Dis. 88:223.

18. Xiao, C. L., Rogers, J. D., Kim, Y. K., and Liu, Q. 2005. Phacidiopycnis washingtonensis - a new species associated with pome fruits from Washington State. Mycologia 97:464-473. 